\title{
Interdisciplinary Cooperation for a New Era of Textile and Fashion Technology
}

\author{
Byung Hoon Kim* \\ Incheon National University, Republic of Korea
}

*Corresponding author: Byung Hoon Kim, Department of Physics, Incheon National University, Incheon, Republic of Korea.
Received Date: July 19, 2018

Published Date: July 27, 2018

\section{Editorial}

Textile and fashion industries are indivisible relation. They are closely interacting components like an organism. The development of textile technology results in the progress of fashion industry and vice versa. Moreover, both are inseparable with natural science, social science, and the arts. In this regard, keeping up with a fashion trend, the researches for dyeing, color, fiber, and textile finishing and treatment have been rigorously performed.

Noteworthy is that the textile industry has gradually increased. It has been expected that the textile industry will grow up to 15 billion dollars in 2019. To dominate this market in advance, a differentiated textile has to be developed with the reflection of the fact that people have been dreaming of a better life, a healthy and convenient life. The flexible and portable electronic devices for the wearable healthcare electronics and wearable computer can make the dream come true. In this light, people have focused on not only the electronic textiles (e-textiles) and smart textiles but also advanced materials that can be used to fabricate e-textiles and smart textiles. Chemists, material scientists, and physicists have suggested the novel approaches to fabricate the textiles. Onedimensional carbon nanotube (CNT) and two-dimensional van der Waals materials such as graphene and graphene oxide (GO) have been considered as one of the candidates for e-textiles because carbon-based materials is highly conductive and light in weight.

For CNT, electronic yarn (e-yarn) was obtained from multi-wall carbon nanotube forest synthesized by chemical vapor deposition method. CNT electronic fabrics were also obtained from the forest using sticky paper such as post-it. Guest materials such as $\mathrm{MgB}_{2}$ and $\mathrm{LiFePO}_{4}$ were easily introduced in the CNT e-yarns. Hence e-yarns for superconductivity and Li-ion battery were synthesized. In the case of graphene-based textiles, GO suspension was put into the pipe and then the pipe was heated. As a result, variously shaped e-textiles could be obtained. Reduced GO coated nylon, polyester, cotton, silk, and spider web were also introduced as the e-textiles. In addition, the high thermally-durable e-textiles can be coated various materials such as semiconducting and superconducting materials was achieved using commercial silk itself with applying axial tension during the thermal treatment.

All e-textiles I have introduced so far were fabricated by a fusion of many disciplines such as chemistry, materials science, and physics. This interdisciplinary cooperation including social science and arts will be the driving force for a new era of fashion and textile industry. Journal of Textile Science and Fashion Technology envisions the integrated disciplines by dealing with the various fields related to not only textile science and fashion technology but also natural and materials sciences. Furthermore, the Journal of Textile Science and Fashion Technology will provide the agora for the breakthrough idea and innovative technology of lots of disciplines whatever helps for the development of textile and fashion technology. Now, we take the first step. 